\title{
INFALL OF HVC'S AND THE ORIGIN OF HI SUPERSHELLS
}

\author{
I.F. MIRABEL \\ Service D'Astrophysique, CEA-CEN. Saclay, \\ 91191 Gif sur Yvette, FRANCE
}

\begin{abstract}
Surveys for atomic hydrogen at very high velocities $(|V| \geq 140 \mathrm{~km}$ $\mathrm{s}^{-1}$ ) in the galactic center and anticenter regions of the sky reveal a net inflow of gas toward the Milky Way. In the anticenter, the collisions of infalling clouds with galactic material trigger the most energetic structural disturbance in the Galaxy, the "anticenter supershell".
\end{abstract}

\section{INTRODUCTION}

Oort (1970) envisioned for the first time that the penetration into the Galaxy of extragalactic neutral gas with Very High Velocities (VHVC's; $|V| \geq 140 \mathrm{~km} \mathrm{~s}^{-1}$ ) could trigger the formation of High Velocity Clouds at lower velocities (HVC's; 80 $\mathrm{km} \mathrm{s}^{-1} \leq|V| \leq 140 \mathrm{~km} \mathrm{~s}^{-1}$ ). Some years later, Tenorio-Tagle (1980) proposed that the collective action of infalling HVC's subsequently causes the large-scale disturbances at lower velocities observed by Heiles (1979) as HI supershells. To avoid the complications implied by models of differential galactic rotation, I consider only VHVC's in the center and anticenter regions of the sky, namely, those clouds with truly anomalous motions that have not interacted with galactic material and therefore, do not participate in Galactic rotation. A review on infall of neutral gas toward the Milky Way was published in the Proceedings of the IAU Colloquium No 120 (Mirabel, 1989); the observational evidences of this phenomenom were presented in a series of publications (Mirabel and Morras 1984, 1990, and references therein).

\section{EVIDENCE FOR INFALL OF VERY HIGH VELOCITY CLOUDS}

The main difficulties regarding the question of the infall of neutral gas toward the Galaxy reside in two unknowns: the distance to the HI gas clouds, and the effects of differential galactic rotation on the observed radial velocities. The latter difficulty can be overcome by selecting data only from regions of the sky where the line-of-sight component of the solar motion about the galactic center is small. 
Surveys of high velocity $\mathrm{HI}$ in the galactic center and anticenter provide compelling evidence for the galactic accretion of $\mathrm{HI}$ at Very High Velocities. More than $99 \%$ of the VHV gas in the anticenter region has negative velocities (Hulbosch and Wakker, 1988 and references therein). In addition, Mirabel and Morras (1984) found that in the inner Galaxy, $84 \%$ of the VHV gas has negative velocities. The striking preponderence of clouds with the same extreme inward motions in the center and anticenter is the strongest evidence for a high velocity inflow of gas toward the Milky Way.

In the most simple infall scenario the infalling clouds are likely to move preferentially toward the central regions of the Galaxy. In this context, most of the VHV gas seen in the direction of the inner Galaxy must be at distances greater than 10 kpc from the Sun, beyond the galactic center. Hence, for a given physical size, the clouds detected in the inner Galaxy should appear smaller than their counterparts in the anticenter. Maps by Mirabel and Morras (1984) show that in fact the clouds in the inner Galaxy are about 5 times smaller than clouds in the outer Galaxy.

\section{CLOUD-MILKY WAY COLLISIONS AND THE ORIGIN OF HI SUPERSHELLS}

A natural consequence of the high velocity infall of neutral gas must be the collision with Milky Way gas at high z. Oort (1970) had proposed that most high velocity streams of HI with moderate velocities $\left(|V| \leq 140 \mathrm{~km} \mathrm{~s}^{-1}\right)$ are galactic clouds which have been set in motion by gas of extragalactic origin. However, no direct observational evidence for the physical interaction between extragalactic VHVC's and galactic material had been found. Physical associations between gas at VHV and Milky Way gas may be scarce because once the supershell is formed, the impinging cloud should have been shocked and incorporated into the accelerated structures.

The observations by Mirabel and Morras (1990) support the idea by TenorioTagle (1981) that HI supershells result from the interaction between infalling clouds at very high velocities and galactic material. Their observations show the physical association between a stream of impinging high-velocity clouds at $-200 \mathrm{~km} \mathrm{~s}^{-1}$ and the most remarkable HI supershell in the anticenter of the Galaxy (Heiles, 1979).

Taking the Galaxy as a whole, the energy injected by VHVC's will be about $2 \times 10^{47}$ ergs $\mathrm{yr}^{-1}$, only $1 \%$ of the energy injected by supernovae of $10^{51} \mathrm{ergs}$ exploding at a rate of $1 / 50 \mathrm{yr}^{-1}$. However, outside the solar circle, the energy due to the infall of gas may be relatively important, since the supernova rate decreases with galactocentric distance. Comparatively, few molecular gas clouds and massive stars populate the outer regions of the Galaxy, and in those regions, supernovae explosions may not dominate completely the energy balance of the interstellar medium. 


\section{REFERENCES}

Heiles, C. (1979) Astrophys. J. 55, 585

Hulsbosch, A.N.M., Wakker, B.P. (1988) Astron. Ap. Supp 75, 191

Kulkarni, S.R., Mathieu, R. (1986) Ap. Space Sci. 118, 531

Mirabel, I.F. (1989) in Structure and Dynamics of the Interstellar Medium, eds. G. Tenorio-Tagle, M. Moles, J. Melnick, p. 396

Mirabel, I.F., Morras, R. (1984) Astrophys. J., 279, 86

Mirabel, I.F., Morras, R. (1990) Astrophys. J. 356, 130

Oort, J.H. (1970) Astr. Ap. 7, 381

Tenorio- Tagle, G. (1980) Astron. Ap. 88, 61 


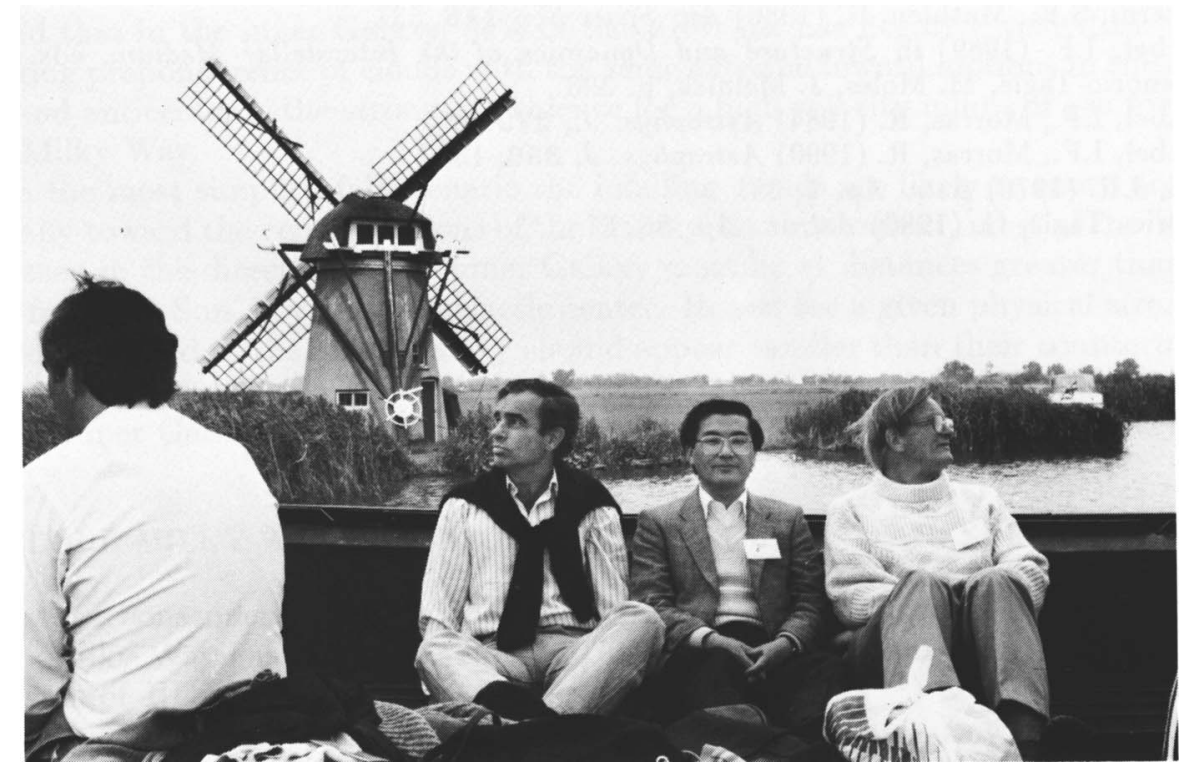

